DOI: https://doi.org/10.46296/ig.v3i6.0017

\title{
RESULTADOS DEL PROCEDIMIENTO DE DIGESTIÓN ANAERÓBICA PARA EL TRATAMIENTO DE RESIDUOS SÓLIDOS URBANOS EN ECUADOR.
}

\section{RESULTS OF THE ANAEROBIC DIGESTION PROCEDURE FOR THE TREATMENT OF URBAN SOLID WASTE IN ECUADOR.}

\author{
Muñoz-Menéndez María Belén ${ }^{1}$; Contreras-Moya Ana Margarita2; Santos-Herrero Ronaldo \\ Francisco3; Rosa-Domínguez Elena Regla ${ }^{4}$; Cárdenas-Ferrer Teresa Margarita ${ }^{5}$ \\ 1 Universidad Laica Eloy Alfaro de Manabí, ULEAM. Manta, Ecuador. \\ beleta_1983@hotmail.com. https://orcid.org/0000-0002-1705-2259. \\ ${ }^{2}$ Universidad Central Martha Abreu de las Villas. Santa Clara, Cuba. \\ anama@uclv.edu.cu. https://orcid.org/0000-0001-9374-9376. \\ ${ }^{3}$ Universidad Central Martha Abreu de las Villas. Santa Clara, Cuba. \\ ronaldo@uclv.edu.cu. https://orcid.org/0000-0002-5009-2084. \\ ${ }^{4}$ Universidad Central Martha Abreu de las Villas. Santa Clara, Cuba. \\ erosa@uclv.edu.cu.https://orcid.org/0000-0002-5371-0976. \\ ${ }^{5}$ Universidad Central Martha Abreu de las Villas. Santa Clara, Cuba. \\ teresam@nauta.cu. https://orcid.org/0000-0003-2054-3136.
}

\begin{abstract}
Resumen
Los problemas de control y estabilización de los sistemas convencionales de digestión anaerobia han llevado a nuevas soluciones tecnológicas, entre estas la separación de fases, que permite la optimización de la acidogénesis y metanogénesis por separado. La primera fase ha sido poco estudiada y en esta se obtienen, por fermentación, hidrógeno y ácidos grasos volátiles, cuyas aplicaciones hacen que el proceso de fermentación acidogénica tome importancia y sea campo de estudio; presentándose como una plataforma que consolida el concepto de biorefinería en lugar de una etapa de tratamiento convencional. Este trabajo tiene el objetivo de presentar los resultados del proceso de digestión anaeróbica de residuos sólidos urbanos de Manta, Ecuador. A partir de los resultados de caracterización, que aportan la generación percápita y evidencian que en la composición predomina la fracción orgánica, se justifica el estudio de aprovechamiento de dicha fracción mediante digestión anaeróbica.
\end{abstract}

Palabras clave: Residuos sólidos urbanos; digestión anaeróbica; fases separadas; análisis de ciclo de vida.

\begin{abstract}
The control and stabilization problems of conventional anaerobic digestion systems have led to new technological solutions, among them phase separation, which allows the optimization of acidogenesis and methanogenesis separately. The first phase has been little studied and in this phase hydrogen and volatile fatty acids are obtained by fermentation, the applications of which make the acidogenic fermentation process become important and a field of study; presenting itself as a platform that consolidates the biorefinery concept instead of a conventional treatment stage. This work aims to present the results of the anaerobic digestion process of urban solid waste in Manta, Ecuador. From the characterization results, which provide the per capita generation and show that the organic fraction predominates in the composition, the study of the use of said fraction by anaerobic digestion is justified.
\end{abstract}

Keywords: Solid urban waste; anaerobic digestion; separate phases; Life cycle analysis.

Información del manuscrito:

Fecha de recepción: 30 de abril de 2020

Fecha de aceptación: 04 de julio de 2020

Fecha de publicación: 10 de julio de 2020 


\section{Introducción}

En la actualidad a nivel mundial, se destaca la generación y disposición final de los residuos sólidos como un tema relevante $y$ de creciente interés, esto porque el crecimiento demográfico e industrial hace que diariamente se arrojen millones de toneladas a las superficies terrestre y acuática, sin ningún tipo de tratamiento ni manejo previo, produciéndose una grave contaminación que implica consecuencias irreversibles.

Según investigadores del Banco Mundial, (Hoornweg y Bhada-Tata 2012), para el año 2025 se espera que la generación de Residuos Sólidos Urbanos (RSU) tienda a duplicarse. Entre las causas de este incremento, además del alto crecimiento poblacional, se mencionan los hábitos de consumo en países industrializados, así como los cambios en las costumbres de consumidores que habitan los países en vías de desarrollo.

Todos los recursos naturales son afectados por los procesos de manejo y disposición final de los residuos sólidos (Jaramillo y Zapata, 2008), ya que la gestión inadecuada de estos afecta todas las actividades, personas y espacios.

La generación de residuos tiene una triple repercusión ambiental, contaminación, desperdicio de recursos y la necesidad de espacio para su disposición. Por lo que las implicaciones que los mismos plantean sobre la contaminación ambiental y el agotamiento de los recursos naturales, hace que sea esencial la búsqueda de caminos para su gestión correcta desde el punto de vista ambiental, económico y social. Una gestión que aborde el ciclo completo de los residuos sólidos, desde la generación, recolección y transporte hasta la disposición final, el tratamiento y su eventual reutilización y aprovechamiento, conlleva claros beneficios para la salud humana (Tenorio, 2008; ONU-Hábitat, 2012).

La gestión integral de los residuos sólidos busca ser compatible con las preocupaciones ambientales y la salud pública, se enmarca en la filosofía del desarrollo sostenible. En diferentes países la jerarquización de tales alternativas es similar y se agrupa como: prevención (minimización y reducción en la fuente), valorización, reutilización, 
reciclaje y compostaje, recuperación de energía (digestión anaerobia, incineración, etc.) y disposición final en rellenos sanitarios (Barradas, 2009; Castellanos, 2017). Las tecnologías implicadas inciden en los sistemas productivos, ya que se hace necesario producir más con menos recursos. Considerando lo anterior, la actual jerarquía de gestión de residuos puede verse como un menú de opciones de recuperación, entre la prevención y la disposición final y esto es coincidente con lo formulado por diferentes autores (Soto, 2014; Mandujano, 2001; Castellanos, 2017).

Se ratifica el valor del tratamiento, ya que modifica las características físicas, químicas o biológicas de los residuos, para aprovecharlos, estabilizarlos o reducir su volumen, antes de la disposición final, considerando que la gestión adecuada de los RSU requiere tratar por separado la FORSU y la fracción inorgánica.

De forma general, los RSU orgánicos no deben ser enviados a los rellenos sanitarios (RS) y los inorgánicos, deberán ser separados de forma adecuada para recuperar los residuos reciclables antes de la disposición en RS. De esta forma, a los RS llegarán los RSU inorgánicos no reciclables y algunos orgánicos no aprovechables. La generación de metano de ese RS, al que no se le "alimenta" con residuos orgánicos, será más limitada que en otros, pero su capacidad para recibir residuos podrá ser mucho mayor (Escalona, 2014; Romero-Paredes, 2013).

El proceso de digestión anaerobia en fases separadas es un esquema novedoso que mantiene dos reactores en serie, en los que se llevan a cabo las fases de acidogénesis y metanogénesis, respectivamente con el objetivo de conseguir un tiempo de retención global inferior al de un único reactor.

En la etapa acidogénica (fermentación oscura) se genera un biogás con alto contenido en hidrógeno, lo que tiene especial interés ya que el hidrógeno se considera el vector energético del futuro próximo y su producción a partir de la degradación de residuos orgánicos presenta un especial interés (Angeriz, 2018), ya que, como se mencionó anteriormente, reciclar tomado el residuo como materia prima es la estrategia más 
afín al principio de sustentabilidad y las biorrefinerías constituyen una de las aplicaciones más avanzadas de la misma.

La biomasa es el mayor contribuyente a la generación de energías renovables (Bentsen, 2019) y según BIOPLAT y SusChem-España (2017) y Chandra et al. (2019), se refiere a la fracción biodegradable de los productos y residuos de origen biológico procedentes de diferentes actividades, así como la fracción biodegradable de los residuos industriales y municipales (FORSU).

El aprovechamiento de la biomasa como materia prima, para la obtención de productos y energía, presenta grandes ventajas pues constituye una fuente renovable, permite tener un control de desechos y reduce la contaminación al disminuir la emisión de gases contaminantes (Agrela et al, 2019).

Para eliminar o disminuir los daños que los RSU provocan se utilizan diferentes métodos de tratamiento entre los que se destaca la digestión anaerobia como uno de los procesos más atractivos en la actualidad. La digestión anaeróbica es un proceso que convierte la materia orgánica en una mezcla gaseosa compuesta principalmente por metano y dióxido de carbono. Los problemas en el control y estabilización de los sistemas convencionales de digestión anaerobia han llevado al desarrollo de nuevas soluciones tecnológicas, entre estas la separación de fases, que implica una configuración de reactores separados; una primera fase para la hidrólisis y acidogénesis y una segunda para la acetogénesis y metanogénesis, conectados en serie, permitiendo la optimización de cada proceso por separado. La primera fase ha sido poco estudiada, la cual consiste en la conversión de compuestos complejos, como los aminoácidos, glucosa y ácidos grasos de cadena larga, por medio de la fermentación, en hidrógeno y ácidos grasos volátiles (AGV) (acético, butírico, propiónico y valérico). La mayoría de los AGV para uso industrial que se producen en el mundo, se obtienen a partir de combustibles fósiles, mediante de síntesis química. Por lo tanto, la fermentación acidogénica se presenta como una plataforma que consolida el concepto de biorefinería 
en lugar de una etapa de tratamiento convencional.

Las diferentes aplicaciones del hidrógeno y los AGV hacen que el proceso de fermentación acidogénica tome importancia y sea campo de estudio.

Actualmente se han desarrollado diferentes estudios de producción de biogás a partir de numerosas materias primas, aún insuficientes en el caso de la fracción orgánica de los residuos urbanos (FORSU) y sobre todo en el caso de la digestión anaerobia en fases separadas. El estudio y utilización de estos métodos brinda la posibilidad de tratar un residuo que puede ser contaminante para el medio ambiente, reciclarlo y convertirlo en productos de alto valor agregado.

Escamilla-Alvarado et al. (2012a) reportaron que una serie de procesos de fermentación oscura digestión anaeróbica metanogénica (FO-DA) de la FORSU, que ellos crearon $(\mathrm{H}-\mathrm{M}), \quad(\mathrm{H}$ : la fase de producción de hidrógeno, M: la fase de producción de metano) en procesos termofílicos y mesofílicos, fueron en promedio 76 y $42 \%$ superior, en términos de potencial energético, que el bioreactor metanogénico solo. Además, Escamilla-Alvarado et al. (2012b, 2013a) reportaron otras mejoras logradas en la fermentación de hidrógeno de la serie de procesos FO-DA de la FORSU.

En la literatura científica se observa un interés por la valoración de las cadenas de valor basadas en biomasa, cuya sostenibilidad es principal para la implantación de biorefinerías, ya que los productos de estas deben mostrar menores impactos que los productos convencionales (De Jong y Jungmeier, 2015; Saraiva, 2017; Cherubini y Strømman, 2011; Ivanov et al., 2015; Zhang, 2008). Las metodologías más comunes que aportan valoraciones adecuadas son el análisis de ciclo de vida (ACV) y análisis costo beneficio. De aquí que una valoración científicamente fundamentada, basada en ACV de nuevos productos basados en biomasa es beneficioso para la toma de decisiones (Lindorfer, et al. 2019).

Coincidiendo con lo anterior y otros autores, Bovea (2016) plantea la metodología de ACV como la mejor herramienta para evaluar el desempeño medioambiental de 
sistemas de gestión de residuos, ya que permite evaluar, desde una perspectiva global, todos los impactos ambientales que ocasiona la gestión integral de los residuos (Arena et al. 2003, Mc Dougall et al. 2001, Laurent et al. 2014).

El desarrollo de estudios experimentales de la degradación anaeróbica de la fracción orgánica biodegradable presente en los RSU, explica desde el punto de vista teórico experimental la realidad biológica de estos tipos de procesos, según las variables que influyen en este, para a partir de estos modelar y simular el proceso.

El presente trabajo tiene como objetivo realizar un estudio para la evaluación del proceso de digestión anaeróbica de los residuos sólidos urbanos de Manta con énfasis en los productos con alto valor agregado que se pueden obtener en el proceso en fases separadas.

\section{Método}

2.1. Estudio de los parámetros operacionales del proceso de digestión anaeróbica de la FORSU en fases separadas.
A partir de los resultados de la gestión de RSU en Manta y del estudio de las tecnologías disponibles para la transformación y/o aprovechamiento de los mismos, se realiza un análisis técnico y ambiental de la alternativa a recomendar, como la más adecuada desde el punto de vista de la sostenibilidad (Elite Consultor, 2011).

La alternativa incluye una planta de transferencia y clasificación de los residuos, reciclaje de los $\mathrm{RSI}$, tanto como sea posible, digestión anaeróbica de la FORSU mediante fases separadas (acidogénesis y metanogénesis) y relleno sanitario.

\section{Análisis de la alternativa.}

Para el análisis de la alternativa se considera la generación de RSU proyectada en Manta para el año 2021, que es de aproximadamente 250 t/d (Elite Consultor, 2011).

En la figura 1 aparece el diagrama de la alternativa que parte de la recogida selectiva de los $R S U$ y la entrada en una planta de transferencia y clasificación, donde se separan los RSI, la FORSU y otros que no se incluyen en estas 
categorías. De la fracción inorgánica se separan los que pueden ser reciclados y los restantes, junto con los denominados "otros" y parte de la FORSU se disponen en el RS; mientras los RSI y FORSU con características adecuadas son enviados a la empresa comercializadora y proceso de digestión anaeróbica respectivamente.

Se consideran potencialmente reciclables 58,05 t de $\mathrm{RSI}$, que, según los resultados de caracterización y criterios para el establecimiento de la alternativa, se corresponden con 26,38 $\mathrm{t}$ de plástico, 22, 93 t de papel y cartón, $4,63 \mathrm{t}$ de vidrio y $4.13 \mathrm{t}$ de metal.

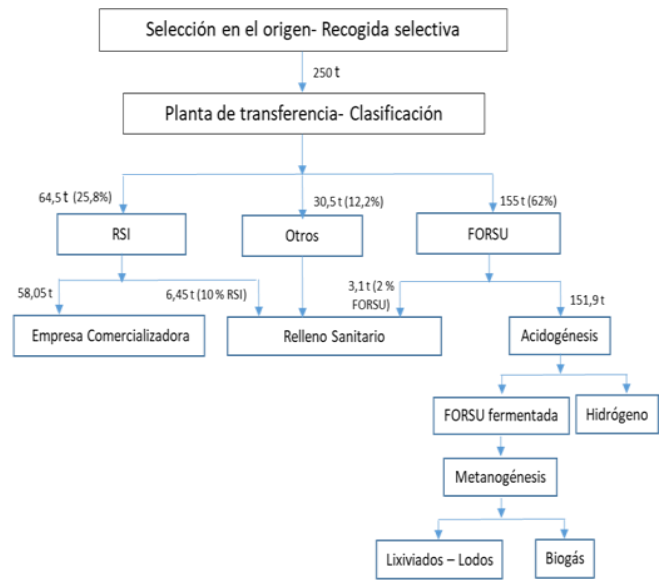

Figura 1. Diagrama de la Alternativa

En la primera fase de la digestión anaeróbica ocurre la hidrólisis y acidogénesis y el efluente de este digestor constituye la alimentación al otro digestor donde ocurre la segunda fase, o metanogénesis.

Como se explicó, los resultados alcanzados en este tipo de procesos demuestran que con la separación de fases es posible disminuir el tiempo de retención, lograr mejor rendimiento en la eliminación de SV y mejor poder calórico en el biogás. Además, el tratamiento de los RSU en unión con los lodos de depuradoras de aguas residuales presenta ventajas en la obtención del gas combustible y las características fertilizantes del efluente (Mandujano, 2001).

Este nuevo proceso tiene otras ventajas, ya que permite obtener simultáneamente hidrógeno y biogás; también se pudieran producir otros productos de alto valor agregado a partir de la FORSU fermentada. La tecnología necesaria, de acuerdo con el esquema que se muestra en la figura 2, está disponible a escala industrial, la misma que ya se está aplicando para la digestión anaeróbica convencional, pero con algunas modificaciones, por lo que se requiere establecer las condiciones de operación adecuadas (EscamillaAlvarado et al., 2011). 


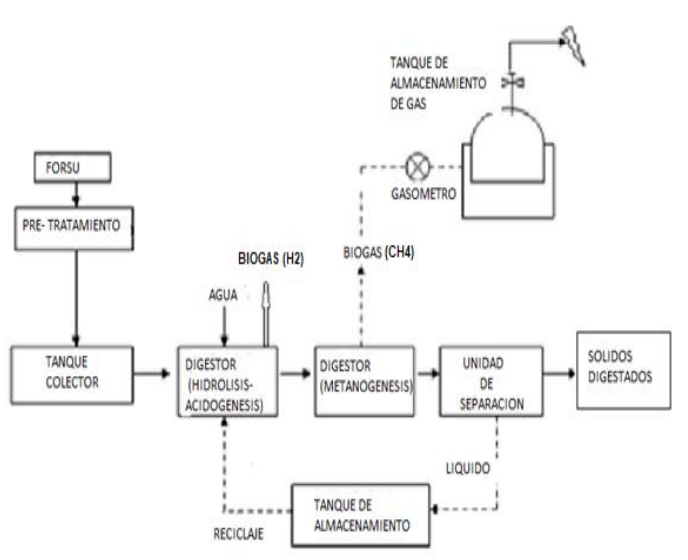

Figura 2. Proceso de digestión anaeróbica en fases separadas.

El pre- tratamiento consiste en la trituración hasta diámetros de 0,3- 5 $\mathrm{mm}$ en condiciones húmedas, a las que se les realizó análisis de ST, SV y $\mathrm{pH}$.

Las condiciones de operación para el tratamiento de la FORSU mediante digestión anaeróbica en fases separadas se establecen mediante el procedimiento experimental que se describe.

\subsection{Diseño del procedimiento experimental}

La simulación a nivel de laboratorio del proceso consta de los siguientes pasos:

1. Preparación de la FORSU:

- Recepción,

- Selección,

- Separación,

- Reducción de tamaño
2. Adición de humedad y de nutrientes:

- Adición de agua residual proveniente de depuradora de residuales urbanos.

- Mezclado,

- Ajuste de $\mathrm{pH}$,

- Estabilización del proceso

3. Evaluación- control del proceso y recogida de los productos:

- Medición de los parámetros de evaluación- control, con la frecuencia establecida,

- Captura y almacenamiento de los componentes gaseosos,

- Deshidratación y evacuación de los fangos digeridos.

El sistema en fases cuenta con dos biodigestores. Se utilizan los digestores de vidrio existentes en el laboratorio de reactores de la facultad de Química Farmacia, los cuales tienen un volumen de trabajo de diez litros. El sistema cuenta con regulación de temperatura mediante un termostato de circulación Haber, el cual está diseñado para usos en laboratorios y en la industria. Dispone de una pantalla gráfica que permite leer informaciones $y$ 
visualizar

transcursos

de

temperatura.

Para este experimento se prepara en el laboratorio una mezcla de FORSU (que represente aproximadamente la composición en carbohidratos, proteínas, etc. de la FORSU original, a partir de restos de comida, carnes, verduras, frutas, cascaras de vegetales, postres, etc y se caracteriza.

La reducción de tamaño se realiza mediante la molienda de la FORSU a un tamaño de partícula de $5 \mathrm{~mm}$ aproximadamente, en condiciones húmedas.

Una vez reducido el tamaño de los residuos seleccionados, se procede a hidratar con la cantidad de residual establecida, ya que se estudia la codigestión con agua de una planta de tratamiento anaerobia de aguas residuales o una laguna anaeróbica.

La caracterización física química de los sustratos: agua residual, FORSU y mezcla de agua-FORSU se realiza mediante los parámetros y métodos que se muestran en las Tablas 1 y 2 .
Tabla1. Parámetros utilizados para la caracterización del residual y la mezcla agua- FORSU.

\begin{tabular}{|l|l|l|}
\hline \multicolumn{1}{|c|}{ Parámetro } & Unidad & \multicolumn{1}{c|}{ Método } \\
\hline Sólidos Totales & $\mathrm{Kg} / \mathrm{m}^{3}$ & Standard \\
(ST) & $\mathrm{Kg} / \mathrm{m}^{3}$ & $\begin{array}{l}\text { methods } \\
\text { SPHA, } \\
\text { Sólidos Totales }\end{array}$ \\
Volátiles (STV) & & 2005) \\
\hline
\end{tabular}

Tabla 2. Parámetros utilizados para la caracterización de la FORSU.

\begin{tabular}{|l|l|l|}
\hline Parámetro & Unidad & \multicolumn{1}{|c|}{ Método } \\
\hline Materia & $\%$ & Técnicas \\
orgánica, & $\mathrm{Kg} / \mathrm{m}^{3}$ & $\begin{array}{l}\text { Analíticas } \\
\text { estandarizadas } \\
\text { Sólidos }\end{array}$ \\
Volátiles & & \\
\hline
\end{tabular}

Se implementa el sistema en dos fases en rango mesofílico, cuyo valor óptimo de temperatura es de $30^{\circ} \mathrm{C}$ a $33^{\circ} \mathrm{C}$ y se evalúa su eficiencia en el tratamiento mediante monitoreo y análisis de los parámetros operacionales y de control establecidos (Mandujano, 2001).

Según Montes (2008), la concentración de sólidos totales en digestores anaerobios debe estar entre 22-28 kg/m³, para ser considerados de Alta Carga, valor dado por Tchobanoglous (2004). Por lo que, la concentración adecuada de FORSU para preparar la alimentación al digestor está entre 70 y $90 \mathrm{~g}$ de FORSU/L.

La determinación de los parámetros fisicoquímicos para la 
caracterización y operación del sistema se realiza según se describe en la tabla 3.

Tabla 3. Parámetros utilizados para la operación y control del sistema.

\begin{tabular}{|l|l|l|}
\hline \multicolumn{1}{|c|}{ Parámetro } & Unidad & \multicolumn{1}{c|}{ Método } \\
\hline Temperatura & ${ }^{\circ} \mathrm{C}$ & Termómetro \\
\hline $\mathrm{pH}$ & - & $\mathrm{pH}$ metro \\
\hline $\begin{array}{l}\text { Sólidos } \\
\text { Totales }\end{array}$ & $\mathrm{Kg} / \mathrm{m}^{3}$ & $\begin{array}{l}\text { Standard } \\
\text { methods } \\
\text { (APHA, 2005) }\end{array}$ \\
\hline $\begin{array}{l}\text { Sólidos } \\
\text { Volátiles }\end{array}$ & $\mathrm{Kg} / \mathrm{m}^{3}$ & $\begin{array}{l}\text { Standard } \\
\text { methods } \\
\text { (APHA, 2005) }\end{array}$ \\
\hline Biogás & $\mathrm{L} / \mathrm{d}$ & $\begin{array}{l}\text { Desplazamiento } \\
\text { de agua }\end{array}$ \\
\hline
\end{tabular}

Posteriormente con los sustratos ya acondicionados, preparar la mezcla residual- FORSU, a esta mezcla se le determina: ST y SV con la finalidad de establecer las condiciones del sustrato de alimentación.

\subsection{Estabilización}

Después de la inoculación el sistema entra a un periodo de aclimatación, para que los microorganismos se adapten al sistema.

La evaluación del sistema se realiza mediante el análisis y monitoreo de los parámetros de la Tabla 3 . Además, se determina la riqueza del hidrógeno y del metano, mediante cromatografía de gases.
En la Tabla.4 se muestran las condiciones de operación de ambas fases, basado en criterios de Montes (2008); Mandujano (2001) y Lagunes-Paredes, et al. (2016).

Tabla 4. Valores de los parámetros de control del sistema en fases.

\begin{tabular}{|l|l|l|}
\hline \multirow{2}{*}{ Parámetro } & \multicolumn{2}{|c|}{ Valor } \\
\cline { 2 - 3 } & $\begin{array}{c}\text { Fase } \\
\text { Acidogénic } \\
\text { a }\end{array}$ & $\begin{array}{c}\text { Fase } \\
\text { Metanogéni } \\
\text { ca }\end{array}$ \\
\hline $\begin{array}{l}\text { Temperatura } \\
\left({ }^{\circ} \mathrm{C}\right)\end{array}$ & $30 \pm 2$ & $31 \pm 2$ \\
\hline $\mathrm{pH}$ & $5,5-6,5$ & $3,75-4,3$ \\
\hline $\begin{array}{l}\text { Tamaño de } \\
\text { partícula } \\
\text { (mm) }\end{array}$ & $0,3-5$ & \\
\hline $\begin{array}{l}\text { Concentraci } \\
\text { ón de ST } \\
\left.\text { (kg ST/m }{ }^{3}\right)\end{array}$ & $22-28$ & \\
\hline TRH (d) & $2-3$ & $7-15$ \\
\hline $\begin{array}{l}\text { Volumen de } \\
\text { trabajo }(\mathrm{L})\end{array}$ & 10 & 10 \\
\hline
\end{tabular}

Se propone un diseño experimental tipo factorial $2^{3}$ con dos replicas, con los niveles de variables que se muestran en la Tabla 5.

Tabla 5. Valores de los niveles de las variables de estudio del diseño factorial.

\begin{tabular}{|l|l|l|l|}
\hline \multicolumn{1}{|c|}{ Variable } & $\begin{array}{c}\text { Abreviatu } \\
\text { ra }\end{array}$ & $\begin{array}{c}\text { Nivel } \\
\text { Inferi } \\
\text { or (-) }\end{array}$ & $\begin{array}{c}\text { Nivel } \\
\text { superi } \\
\text { or (+) }\end{array}$ \\
\hline $\begin{array}{l}\mathrm{pH} \text { inicial } \\
\text { de la } \\
\text { mezcla }\end{array}$ & $\mathrm{X} 1$ & 5,5 & 6,5 \\
\hline $\begin{array}{l}\text { Concentraci } \\
\text { ón inicial de } \\
\text { sustrato (kg } \\
\text { ST/m }{ }^{3} \text { ) }\end{array}$ & $\mathrm{X} 2$ & 22 & 28 \\
\hline $\begin{array}{l}\text { Tiempo de } \\
\text { retención } \\
\text { hidráulico } \\
\text { total }\end{array}$ & $\mathrm{X} 3$ & 10 & 15 \\
\hline
\end{tabular}

Fuente: Elaboración propia a partir de (Montoya-Pérez y Duran- Herrera, 2017; Mandujano, 2001). 
Variables respuesta: remoción de ST y remoción de STV, rendimiento en la producción de hidrógeno y en la producción de metano.

En la tabla 6 se muestra la matriz del diseño.

Tabla 6. Matriz de diseño.

\begin{tabular}{|l|l|l|l|l|l|l|}
\hline \multirow{2}{*}{$\begin{array}{l}\text { Experim } \\
\text { ento }\end{array}$} & \multicolumn{2}{|l|}{$\begin{array}{l}\text { Condicion } \\
\text { es X1 }\end{array}$} & \multicolumn{2}{l|}{$\begin{array}{l}\text { Condicion } \\
\text { es X2 }\end{array}$} & \multicolumn{2}{l|}{$\begin{array}{l}\text { Condicion } \\
\text { es X3 }\end{array}$} \\
\cline { 2 - 8 } & $\begin{array}{l}\text { Niv } \\
\text { el }\end{array}$ & $\begin{array}{l}\text { Val } \\
\text { or }\end{array}$ & $\begin{array}{l}\text { Niv } \\
\text { el }\end{array}$ & $\begin{array}{l}\text { Val } \\
\text { or }\end{array}$ & $\begin{array}{l}\text { Niv } \\
\text { el }\end{array}$ & $\begin{array}{l}\text { Val } \\
\text { or }\end{array}$ \\
\hline 1 & - & 5,5 & - & 22 & - & 10 \\
\hline 2 & + & 6,5 & - & 22 & - & 10 \\
\hline 3 & - & 5,5 & + & 28 & - & 10 \\
\hline 4 & + & 6,5 & + & 28 & - & 10 \\
\hline 5 & - & 5,5 & - & 22 & + & 15 \\
\hline 6 & + & 6,5 & - & 22 & + & 15 \\
\hline 7 & - & 5,5 & + & 28 & + & 15 \\
\hline 8 & + & 6,5 & + & 28 & + & 15 \\
\hline
\end{tabular}

Con los resultados experimentales se realiza un ACV con el objetivo de identificar las etapas del proceso donde se producen las emisiones con mayor relevancia.

Para el estudio de ACV solo se considera el proceso de digestión anaeróbica de la FORSU, el reciclaje de los RSI y la disposición en relleno sanitario.

Como unidad funcional se toma el tratamiento de la FORSU con características adecuadas para la digestión anaeróbica, producida diariamente en Manta, resultando un flujo de referencia de 151,9 $\mathrm{t}$ de FORSU.

Los datos del inventario de ACV fueron obtenidos de los resultados experimentales y resultados de la literatura. Las entradas y las salidas fueron ajustadas a la unidad funcional. No se llevó a cabo la asignación de cargas ambientales, sino que se extendieron los límites del sistema para considerar los productos evitados por el aprovechamiento de los subproductos.

Para el análisis se utilizó el Software SimaPro de la empresa PRéConsultants, el método de $\mathrm{ReCiPe}$ 2016 v1.1, versión Hierarchist $(H)$ y la base de datos Ecoinvent $\mathrm{v} 3$.

\section{Resultados y discusión}

\subsection{Resultados experimentales.}

En la Tabla 7 se muestran los resultados experimentales de la fase acidogénica, donde aparecen los valores Sólidos Totales iniciales (STi) y en el efluente (STe), los Sólidos Totales Volátiles iniciales (STVi) y en el efluente (STVe), expresados en $\mathrm{mg} / \mathrm{L}$ y los respectivos porcientos de remoción, 
de acuerdo a los valores de $\mathrm{pH}$ y carga orgánica de ST (COST) y de STV (COsv) establecidos, expresados en $\mathrm{kg} \mathrm{ST} / \mathrm{m}^{3} \mathrm{~d}$ y $\mathrm{kg}$ $\mathrm{STV} / \mathrm{m}^{3} \mathrm{~d}$, según la matriz de diseño que aparece en la tabla 6 para un tiempo de retención hidráulico $(\mathrm{TRH})$ de 3 días.

Tabla 7. Resultados experimentales para la fase acidogénica.

\begin{tabular}{|c|c|c|c|c|c|c|c|c|c|c|c|}
\hline & pHi & pHe & COst & COsv & TRH & STi & STe & \%Rem & STVi & STVe & \%Rem \\
\hline 1 & 5.5 & 3.31 & 22 & 19.56 & 3 & 68.7 & 60.7 & 11.64 & 61.14 & 54.73 & 10.48 \\
\hline 2 & 6.5 & 3.86 & 22 & 19.58 & 3 & 68.7 & 62.86 & 8.50 & 61.14 & 55.02 & 10.01 \\
\hline 3 & 5.5 & 3.34 & 28 & 25.98 & 3 & 87.5 & 76.13 & 12.99 & 81.19 & 69.41 & 14.51 \\
\hline 4 & 6.5 & 4.63 & 28 & 25.76 & 3 & 87.5 & 77 & 12.00 & 80.50 & 70.19 & 12.81 \\
\hline 5 & 5.5 & 3.40 & 22 & 19.68 & 3 & 68.7 & 60.52 & 11.91 & 61.53 & 53.96 & 12.30 \\
\hline 6 & 6.5 & 4.26 & 22 & 20.00 & 3 & 68.7 & 61.14 & 11.00 & 62.57 & 55.06 & 12.00 \\
\hline 7 & 5.5 & 3.57 & 28 & 25.48 & 3 & 87.5 & 74.37 & 15.01 & 79.63 & 66.09 & 17.00 \\
\hline 8 & 6.5 & 3.56 & 28 & 25.19 & 3 & 87.5 & 75.25 & 14.00 & 78.75 & 66.70 & 15.30 \\
\hline Prom & 6 & 3.74 & 25 & 22.65 & 3 & 78.1 & 68.4963 & 12.13 & 70.81 & 61.40 & 13.05 \\
\hline Desv St & 0.5 & 0.45 & 3 & 2.96 & 0 & 9.4 & 7.25419 & 1.85 & 9.24 & 6.82 & 2.25 \\
\hline Max & 6.5 & 4.63 & 28 & 25.98 & 3 & 87.5 & 77 & 15.01 & 81.19 & 70.19 & 17.00 \\
\hline Min & 5.5 & 3.31 & 22 & 19.56 & 3 & 68.7 & 60.52 & 8.50 & 61.14 & 53.96 & 10.01 \\
\hline
\end{tabular}

Se observa que en la fase acidogénica se obtienen valores promedio de remoción de Sólidos Totales (ST) de 12, $13 \%$, de Sólidos Totales Volátiles de 13, $05 \%$ y el pH promedio que se alcanza es de 3,74; valor que se considera adecuado para la fase acidogénica. El mayor \% de remoción de ST y STV se obtiene para un valor de $\mathrm{pH}$ de entrada de 5,5; una carga orgánica de 28 kg $\mathrm{ST} / \mathrm{m}^{3} \mathrm{~d}$.

De forma similar, en la Tabla 8 se muestran los resultados experimentales de la fase metanogénica.

Tabla 8. Resultados experimentales para la fase metanogénica.

\begin{tabular}{|c|c|c|c|c|c|c|c|c|c|}
\hline & TRH & STi & STe & $\%$ Rem & STVi & STV & $\%$ Rem & pHi & pHe \\
\hline 1 & 7 & 60.7 & 33.60 & 44.65 & 54.73 & 27.40 & 49.94 & 3.31 & 7.40 \\
\hline 2 & 7 & 62.86 & 37.00 & 41.14 & 55.02 & 29.10 & 47.11 & 3.86 & 7.79 \\
\hline 3 & 7 & 76.13 & 38.40 & 49.56 & 69.41 & 30.40 & 56.20 & 3.34 & 7.68 \\
\hline 4 & 7 & 77 & 40.80 & 47.01 & 70.19 & 32.80 & 53.27 & 4.63 & 7.11 \\
\hline 5 & 12 & 60.52 & 25.40 & 58.03 & 53.96 & 18.20 & 66.27 & 3.40 & 7.04 \\
\hline 6 & 12 & 61.14 & 29.30 & 52.08 & 55.06 & 22.80 & 58.59 & 4.26 & 7.34 \\
\hline 7 & 12 & 74.37 & 25.10 & 66.25 & 66.09 & 16.90 & 74.43 & 3.57 & 7.01 \\
\hline 8 & 12 & 75.25 & 28.40 & 62.26 & 66.70 & 18.50 & 72.26 & 3.56 & 6.91 \\
\hline Prom & 9.5 & 68.50 & 32.25 & 52.62 & 61.40 & 24.51 & 59.76 & 3.74 & 7.29 \\
\hline Desv St & 2.5 & 7.25 & 5.67 & 8.25 & 6.82 & 5.81 & 9.53 & 0.45 & 0.30 \\
\hline Max & 12 & 77.00 & 40.80 & 66.25 & 70.19 & 32.80 & 74.43 & 4.63 & 7.79 \\
\hline Min & 7 & 60.52 & 25.10 & 41.14 & 53.96 & 16.90 & 47.11 & 3.31 & 6.91 \\
\hline & & & & & & & & & \\
\hline
\end{tabular}

En la fase metanogénica, el valor promedio de remoción de ST es de $52,62 \%$ y de STV es de 59,76\%, con un valor promedio de $\mathrm{pH}$ de 7 , 29. El mayor \% de remoción de ST y STV se obtiene para el valor de $\mathrm{pH}$ de entrada de 5,5; carga orgánica de $28 \mathrm{~kg} \mathrm{ST} / \mathrm{m}^{3} \mathrm{~d}$ y TRH de 12 días.

Estos resultados se encuentran en el rango de los obtenidos por otros autores (Mandujano, 2001).

\section{Conclusiones}

Se observa que los principales impactos positivos están dados por el relleno sanitario, por sus efectos en la eutrofización del agua de mar, ecotoxicidad del agua fresca y de mar, así como en la toxicidad 
humana cancerígena y no cancerígena y calentamiento global, producto de las emisiones que se generan, ya que es un relleno sanitario donde no se recolectan el biogás y lixiviados. También se destacan los impactos positivos del tratamiento de la FORSU, por sus efectos en el agotamiento del ozono estratosférico, la eutrofización del agua fresca, escasez de recursos minerales y en menor medida en el consumo de agua, uso del suelo y toxicidad humana cancerígena.

Resultan de gran valor los resultados negativos para la mayoría de las categorías de impacto como resultado del reciclaje de los RSI y la valorización de los productos de la digestión anaeróbica, lo que hace que los mismos sean procesos muy atractivos para la gestión de la FORSU de Manta.

\section{Bibliografía}

Agrela, F., Cabrera, M., Martín M., Zamorano, M., Alshaaer, M. 2019. Biomass fly ash and biomass bottom ash. New Trends in Eco-efficient and Recycled Concrete. Disponible en: https://doi.org/10.1016/B9780-08-102480-5.00002-6.

Angeriz, R. 2018. Producción de bio- hidrógeno por co-digestión anaerobia acidogénica de residuos sólidos urbanos, residuos alimentarios de cocina y lodos de depuradora. Trabajo de tesis. Ciencias tecnológicas, Ingeniería y tecnología del medio ambiente. Universidad de Cádiz. España. 2018.

APHA, "Standard methods for the examination of water and wastewater". American Public Health Association, American Water Works Association, Water Environment Federation. 21st edition, ISBN-13:978-0875530475.

Public Health Association, Washington, USA, 2005.

Arena U., Mastellone M.L. y Perugini F. (2003). Life Cycle Assessment of a plastic packaging recycling system. Int. J. Life Cycle Assess. 8 (2), 92-98.

DOI: 10.1007/BF02978432.

Barradas, A. 2009. Gestión Integral de Residuos Sólidos Municipales. Estado del Arte. Veracruz, México: Instituto Tecnológico de Minatitlán. Extracto de la tesis de Doctor en Ciencias en Ingeniería Ambiental del autor, por la Universidad Politécnica de Madrid, pp. 4-9.

Bentsen, N.S. 2019. Biomass for Biorefineries: Availability and Costs. Biorefinery, pp 37-47. 
Disponible

en: https://doi.org/10.1007/978-3030-10961-5_2.

BIOPLAT y SusChem-España (Plataformas Tecnológicas Españolas de Biomasa para la Bioeconomía y de Química Sostenible). 2017. Manual sobre las Biorrefinerías en España.

http://www.bioplat.org/setup/u pload/modules_docs/content_ URI_4020.pdf.

Bovea, M., Cruz, S., Mercante, I., Coutinho, C., Eljaiek, M., Ibáñez, V. 2016. Aplicación de la metodología de ACV para evaluar el desempeño ambiental de sistemas de gestión de residuos en Iberoamérica., Revista Internacional de Contaminación Ambiental, Vol. 32, No. Especial Residuos Sólidos, 2016, pp. 23-46.

DOI: 10.20937/RICA.2016.32.05.0 3.

Castellanos, S. 2017. Análisis de Ciclo de Vida para los biorresiduos sólidos urbanos generados en Bogotá D.C, Colombia. Trabajo final para optar al título de Magister en Ingeniería

Ambiental.

Departamento de Ingeniería Química y Ambiental. Universidad Nacional de Colombia. Bogotá D.C, Colombia 2017.
Chandra, R., Iqbal, H.M., Vishal, G., Lee, H.S., Nagra, S. 2019. Algal Biorefinery: A Sustainable Approach to Valorize Algal-based Biomass towards Multiple Product Recovery. Bioresource technology, Vol. 278, pp 346359.

Cherubini, F. \& Strømman, A.H. 2011. Life cycle assessment of bioenergy systems: State of the art and future challenges. Bioresource Technology, 102, 437-451.

De Jong, E. \& Jungmeier, G. 2015. Chapter 1 - Biorefinery Concepts in Comparison to Petrochemical Refineries. In: Pandey, A., Höfer, R., Taherzadeh, M., Nampoothiri, K. M. \& Larroche, C. (eds.) Industrial Biorefineries \& White Biotechnology. Amsterdam: Elsevier.

EEscamilla-Alvarado, C.; Ríos-Leal, E.; Ponce-Noyola, M.T.; Poggi-Varaldo, H.M. (2012a). Gas biofuels from solid substrate hydrogenogenicmethanogenic fermentation of the organic fraction of municipal solid waste. Process Biochemiestry 47: 1572-1587.

Escalona, E. 2014. Daños a la salud por mala disposición de residuales sólidos y líquidos en Dili, Timor Leste. Revista Cubana de Higiene y 
Epidemiología, vol. 52, núm. 2, mayo-agosto, 2014, pp. 270-277.

Escamilla-Alvarado, C.; PoggiVaraldo, H.; Ponce-Noyola, M.T. 2011. Producción de hidrógeno y metano como biocombustibles bajo el esquema de biorrefinería. Rev. Ide@s CONACYTEG, Vol. 6, No. 71, Pág. 526-539.

Hoornweg, D. y Bhada-Tata, P. 2012. What a waste. A Global Review of Solid Waste Management. Washington: World Bank. Urban Development Series, March 2012, No. 15. http://www.preparenet.com/sites/default/files/wh at_a_waste2012_final.pdf.

Ivanov, V., Stabnikov, V., Ahmed, Z., Dobrenko, S. \& Saliuk, A. 2015. Production and applications of crude polyhydroxyalkanoatecontaining bioplastic from the organic fraction of municipal solid waste. International Journal of Environmental Science and Technology, 12, 725-738.

Jaramillo, G. y Zapata, L. 2008. Aprovechamiento de los residuos sólidos orgánicos en Colombia. Medellín: Universidad de Antioquia. Recuperado el 9 de marzo del 2014, de http://tesis.udea.edu.co/dspac e/bitstream/10495/45/1/

Aprovechamiento

RSOUenColombia.pdf.

Lagunes-Paredes, Y.; MontesCarmona, M.E.; VásquezMárquez, A. y CárdenasGuevara, G.E. 2016. Evaluación de la generación de metano y la estabilidad del proceso de codigestión de lodos residuales y fracción orgánica provenientes de un centro comercial. Revista de Ciencias Ambientales y Recursos Naturales. Septiembre 2016 Vol.2 No.5 26-35.

Laurent A., Bakas I., Clavreul J., Bernstad A., Niero M., Gentil E., Hauschild M.Z. y Christensen T.H. (2014) Review of LCA studies of solid waste management systems-Part I: Lessons learned and perspectives. Waste Manage. 34 (3):573-88 DOI: $\quad 10.1016 / j . r e s c o n r e c$. 2012.07.003.

Lindorfer, J.; Lettner, M.; Hesser, F.; Fazeni, K.; Rosenfeld, D.; Annevelink, B.; Mandl, M. 2019. Technical, Economic and Environmental Assessment of Biorefinery Concepts. Developing a practical approach for characterization. IEA (International Energy Agency). Bioenergy: Task 42:2019:01. Copyright (c) 2019 IEA Bioenergy. All rights 
Reserved. ISBN: 978-1910154-64-9.

Mandujano, P. 2001. Digestión anaerobia de sólidos en alta concentración. Tesis Doctoral, Universidad Politécnica de Madrid, 2001.

McDougall F., White P., Franke M., Hindle P. 2001. Integrated Solid Waste Management: a Life Cycle Inventory, (Second edition) Copyright (C) 2001 by Blackwell Science Publishing Ltd.

Montes, M.E. 2008. Estudio técnicoeconómico de la digestión anaerobia conjunta de la fracción orgánica de los residuos sólidos urbanos y lodos de depuradora para la obtención de biogás. Tesis doctoral. Departamento de Ingeniería Civil: Ordenación del Territorio, Urbanismo y Medio Ambiente E.T.S. I. de Caminos, Canales y Puertos.

Montoya-Pérez, L; Durán-Herrera, E. 2017.Producción de hidrógeno a partir de la fermentación de residuos agroindustriales de la piña. Tecnología en Marcha. Vol. 30-3. Julio-Setiembre 2017. Pág 106-118.

Romero-Paredes, A. 2013. Gestión integral de residuos sólidos urbanos de la CEDA. Programa para el Desarrollo Bajo en Emisiones de México
(MLED). CONTRATO: AID523-C-11-00001. Tetra Tech ES Inc. Julio 7, 2013. www.mledprogram.org

Saraiva, A. B. 2017. System boundary setting in life cycle assessment of biorefineries: a review. International Journal of Environmental Science and Technology, 14, 435-452.

Soto, J.L. 2014. Alternativas de recogida, tratamiento $y$ eliminación de residuos sólidos urbanos. Trabajo de Grado en Ingeniería de Obras Públicas. Escuela Técnica Superior de Ingenieros de Caminos Canales y Puertos. Universidad de Valencia. 2014

Tchobanoglous, G., Theisen, H. y Vigil, S. 2004. Gestión integral de residuos sólidos. New York: McGraw-Hill.

Tenorio, M. 2008. Diseño de plan de manejo integral de residuos sólidos para plegacol S.A., Pasantía para de Administrador del Medio Ambiente y de los recursos naturales., Universidad Autónoma de Occidente, Colombia, 2008.

Zhang, Y.H.P. 2008. Reviving the carbohydrate economy via multi-product lignocellulose biorefineries. Journal of Industrial Microbiology \& Biotechnology, 35, 367-375. 